\author{
Military Technical College \\ Kobry El-Kobbah, \\ Cairo, Egypt.
}

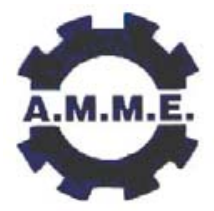

\title{
A NEW TECHNIQUE FOR ACTUATING PARALLEL MANIPULATORS
}

\author{
KHIDIR $^{*}$ E.A., MOHAMED** N.A.N., NOR ${ }^{* *}$ M.J.M. and MUSTAFA*** M.M.
}

\begin{abstract}
This paper presents a new technique of actuating a parallel platform manipulator using shape memory alloy (SMA). This is a type of smart materials that can attain a high strength-to-weight ratio, which makes them ideal for miniature application. The work is divided into two main parts; developing a new SMA actuator and then incorporating the actuator in building the parallel manipulator prototype. The SMA used in this study is a commercial NiTi wire. The SMA wire provides an actuating force that produces a large bending and end displacement. A 3-UPU (Universal-Prismatic-Universal) parallel manipulator using linear SMA actuators was developed. The manipulator consists of a fixed platform, a moving platform and three SMA actuators. The manipulator workspace was specified based on the restrictions due to actuator strokes and joint angle limits. System identification techniques were used to model both heating and cooling processes. An ON/OFF control was performed and the results showed closeness in simulation and experimental results. This study showed that shape memory alloy actuated beam can successfully be used to provide linear displacement. The built prototype indicates the feasibility of using SMA actuators in parallel manipulators.
\end{abstract}

\section{KEY WORDS}

Parallel manipulator, actuator, shape memory alloy

\footnotetext{
* $\quad$ Post Doctoral Fellow, Dpt. of Mech. and Mat. Eng., UKM, Bangi, Malaysia.

** Professor, Dpt. of Mech. and Mat. Eng., UKM, Bangi, Malaysia.

*** Professor, Dpt. of Electrical, Electronic and System, UKM, Bangi, Malaysia.
} 


\section{INTRODUCTION}

Shape memory alloys (SMA) as smart materials have received increasing attention in recent years, especially in the development of innovative engineering systems such as micro-actuators, microvalves, vibration absorbers, etc. They are considered attractive candidate materials for applications in actuators because their dual functionality of sensing and actuating in a single body [1]. The use of these so-called shape memory alloy materials as actuators in robotic applications attempts to take advantage of their large capacities in motion and force transmission. Moreover, the simplicity of their actuation principle and their compatibility with micro system technologies make them very attractive for highly miniaturized and micro-electromechanical systems. Consequently many SMA micro-actuator applications have been tested recently more particularly to build innovative biomedical instruments like endoscopes, forceps, grippers, and prosthetic devices [2].

Conventional actuators used are electrical, hydraulic and pneumatic, but they have difficulties in generating significant forces when their size scaled down. Non-conventional or active materials are materials that deform upon the application of a signal, which may be good alternatives to conventional actuators. There are many types of smart materials, such as SMA alloys, piezoceramics, piezoelectric, electroactive polymers, etc. However, SMA actuators can attain a high strength to weight ratio, which makes them ideal for miniature application [3]. From a mass and volume saving point of view, Hirose et al. [4] have compared the power/weight ratio vs. weight of a particular form of SMA actuator with many other conventional motors.

Shape memory effect refers to the ability of SMA materials to recover a predetermined shape when heated. When a SMA is in the martensite phase at low temperature can be deformed quite easily into a new shape, which it retains. However, when the material is heated so that it is in the austenitic phase at high temperature, the SMA undergoes a change in crystal structure, which causes it to return back to its original shape. Some examples of these alloys are Ag-Cd, Au-Cd, Cu-Sn, Cu-Zn, Ni-Al, Mn-Cu, and Fc-Mn-Si. Under certain conditions the shape of the SMA alloy can be deformed by up to $8 \%$ (such as $\mathrm{NiTi}$ ). Large forces can be generated with these actuators, as the stress limit is more than $250 \mathrm{MPa}$ for working conditions [5]. NiTi alloy show the best mechanical characteristics among the number of alloys, which exhibit a shape memory effect.

SMA alloys are inherently exhibit significant hysteresis in their stress-strain-temperature characteristics, which adversely affects modeling precision control of the structures activated by SMA actuators. SMA alloys are also known for their limited strain. They can deform up to $5 \%$ workable strain. To obtain a higher strain this needs a mechanical amplification that can be obtained using long SMA wires or SMA coils. The long wires occupy long space while the coils suffer from drawback of requiring a larger diameter than necessary. Grant \& Hayward [5] proposed an actuator to achieve mechanical motion amplification avoiding the drawbacks of coils. However, they still used long wires woven in a counter rotating helical pattern around supporting disk, which at the same time needed a strong and high stiffness spring to preload the SMA wires. 
Generally there are two types of manipulators; parallel Manipulators and serial manipulators. So far the application of SMA actuators in robotics is limited to grippers [7] or serial manipulators. The application of SMA actuators in parallel manipulators is not yet documented. Parallel manipulators are robotic devices that differ from the serial manipulators by virtue of their structure. Parallel manipulators composed of two or more kinematic chains that connect a moving platform to a base, where one joint in the chain is actuated and the other joints are passive. This structure allows parallel manipulators to be driven by actuators positioned on or near the base of the manipulator. In contrast, serial manipulators do not have closed kinematic loops and are usually actuated at the joints. This allows the parallel manipulator links to be made lighter.. Stewart Platform is a popular configuration for parallel link manipulator type. It was originally designed by Stewart [8]. Since then, the Stewart platform has also been used for other applications such as milling machines, pointing devices and underground excavation devices. A very interesting aspect of parallel manipulators is that they enable the use of unconventional actuators like, for example, wire with winches [9] or binary actuators that have only two states, fully extended or retracted [10]. Choi et al. [11] used Electro-Rheological (ER) valve actuators for a three-degree of freedom parallel link manipulator. This enable to extend the application area of parallel manipulators but at the same time induces additional constraints on the theoretical problems to be solved (e.g. computing the workspace not only taking into account the limit on the stroke of the actuators but also the preservation of the tension on the wires).

The main objectives of this research is to develop a shape memory alloy (SMA) actuator that is capable of providing a linear displacement; and to incorporate the SMA actuator in building a parallel platform manipulator prototype.

\section{SMA ACTUATORS}

The manipulator is actuated by three linear SMA actuators. The SMA actuator consists of two beams fixed to two Teflon plates of size $40 \mathrm{~mm} \times 35 \mathrm{~mm} \times 8 \mathrm{~mm}$ (Fig. 1). One of the plates is moving and the other is fixed. An SMA wire, of $0.7 \mathrm{~mm}$ diameter, provides an actuating force to produce large bending and hence end displacement of the flexible beam. The wire is fixed eccentrically along a flexible beam dividing it into equally spaced segments. The number of segments used was six, according to the results obtained by Mohamed et. al. [12] at which the strain magnification is maximum. The wires were heat treated, trained and prestrained to $6 \%$ before attached to the beam. The wires were fixed to screws attached to the beam. The screws were electrically insulated by nylon insulating spacers. A shaft fixed to the moving plate and uses the fixed plate as a guide. The fixed part of the shaft is bolted to the moving plate. The beams are connected to the plates through Teflon joints via the end screws to allow the moving plate to push the shaft in forward direction when the beams are deflected as a result of contraction of SMA wire upon heating. The actuator is prevented from twisting by aluminum casing, which is used as a guide for the moving plate. The shaft returns to its initial position after cooling due to the beam stiffness. A compression spring was used because it was found that the beam stiffness alone is not sufficient to return the actuator completely to the initial position. The 
total length of the un-actuated SMA actuator is $250 \mathrm{~mm}$, which is given between the centers of the universal joints at the same prismatic link.

\section{The 3-UPU MANIPULATOR PROTOTYPE}

A prototype was built to provide a proof of the concept for this SMA actuated manipulator. The prototype consists of a moving plate, a base plate and three identical links (SMA actuators). The plates have equilateral triangular shapes of different sizes. The links are connected to the plates with 2-DOF universal joints. Each universal joint is treated as two revolute joints with axes perpendicular to each other and intersecting at a point. The prototype is fully actuated by three SMA actuators and computer controlled. A picture of the manipulator is shown in Fig. 2. The position of each link is measured by a linear variable differential transformer (LVDT).

\section{MANIPULATOR WORKSPACE}

In the field of robotic motion planning, configuration space (workspace) is a useful representational tool. According to the definition of Latombe [13] configuration space is an appropriate space in which the robot is represented by a point, and to which obstacles are mapped. This mapping transforms the problem of planning the motion of a dimensioned object into the problem of planning the motion of a point. It makes the constraints on the motions of the robot more explicit. The workspace can be obtained by performing kinematic analysis.

The kinematic analysis was done using kinematics relations, at which restrictions due to actuator strokes and joint angle limits were introduced. A program in FORTRAN was used to determine the manipulator workspace. At each point the length of the legs and the joint anglesere calculated and verified if they are within the imposed limits. The maximum stroke or displacement for all SMA actuators is around $20 \mathrm{~mm}$. This means the minimum and maximum height of the moving plate from the fixed plate are $213.5 \mathrm{~mm}$ and $236.6 \mathrm{~mm}$, respectively. Fig. 3. to Fig. 7. show horizontal slices of the workspace at different height levels for $\pm \pi / 4\left(45^{\circ}\right)$ universal joints angle constrains. As observed in the figures the workspace increases as the level of slice increases and then decreases again. This is due to the actuator and universal joints limits and constraints.

\section{MANIPULATOR CONTROL}

To achieve the full performance of the manipulator, design of an effective control system is important. The SMA actuators are suffering of uncertainties caused by impossibility of having all its components precisely specified. Therefore, due to the associated difficulties in deriving an accurate analytical model, it was decided that the best route would be to use system identification techniques. Since SMA transformation exhibits a hysteresis effect, in that the transformations on heating and on cooling, the modeling was performed for both heating and cooling separately [14]. An experimental test rig was constructed to evaluate 
the performance of the controller. An ON/OFF controller was designed for the accomplishment of the position tracking control of the proposed manipulator. A schematic diagram of this setup is shown in Fig. 8. The instrumentation components used in the setup consists of three relay switches, three LVDTs, power supply interface card, and a PC. The relay switches are controlled by the difference between the feedback (control variable) from the LVDTs displacement sensors and the desired reference value (set point). A real time code in Borland $\mathrm{C}++$ language was developed to implement the digital control algorithm for the single-input/single-output case. The displacement is controlled by manipulating an electric voltage according to the continuously monitored position.

The manipulator motion tasks require the precise information on the moving plate position. As known the end effecter position can be calculated from the measured link coordinates via the forward kinematics. However, to control the moving plate position, the corresponding link coordinates must be calculated. This is the inverse kinematics. For the desired moving plate position, the links lengths are calculated, and hence the actuators stroke is controlled according to the desired position. As a result the relays will be ON and OFF according to the feedback. The displacement of the actuator is measured with an attached LVDT, and each displacement is controlled independently. Giving the objective points, it was calculated whether the solution exist using the inverse kinematics. The control strategy is simple (Fig. 9): for a given point position ( $x, y, z)$ reference actuator length displacement $d_{i}(i=1,2$ and 3$)$ is computed. If the displacement exceeds $d_{i}+\alpha$ then the voltage $V$ is set as OFF (cooling), and $V$ reset to be ON (heating) when the displacement falls below $d_{i}-\alpha$, where $\alpha$ is the permissible error.

\section{SIMULATION}

Simulink/MATLAB was then used to control simulation as shown in Fig. 10 . The SFunction block is directly linked to the M-file program, which includes both heating and cooling models [14]. It is capable to switch between both models in case of heating and cooling processes with the aid of the input provided by the relay according to the permissible error. The input is the actuators lengths, which is computed for a given point $(x, y, z)$. For a given point $(x, y, z)$ target position, the length of links are computed within the subsystem in Fig. 10. The links length equations are nonlinear. To solve these equations an "Algebraic Constraints" block is used. The block has a property of changing its output in an iterative manner until its input approaches zero. This needs a feedback path from the output to the input. The solution leads to obtaining the actuators lengths and hence the actuators strokes, which need to be controlled. A simulation was run using the Simulink block diagram and the result was verified experimentally using the experimental setup shown in Fig. 8. A sample of the simulation result is shown in Fig. 11. The simulation was run for a given point of $(0,0,215)$. The actuators' stroke are automatically computed and sent to the controller as an input for each link. The controller is an ON/OFF controller of $\pm 0.01 \mathrm{~mm}$ permissible error. The SMA actuators' strokes or the links' displacements were found to be having a similar value of $1.248 \mathrm{~mm}$, which is logical since $x$ and $y$ inputs are of zero values and the moving plate must be kept parallel to the fixed plate. This means the moving plate is moving vertically. 


\section{EXPERIMENTAL RESULTS}

The experimental results were obtained by giving $(x, y, z)$ as a desired position, which will be the input to the system. For a given $(x, y, z)$, the program written in Borland $\mathrm{C++}$ computes the actuators strokes and universal joints angles using the inverse kinematics. These values are checked according to the actuators and the universal joints constraints. If the values are out of the range an invalid entry message will be obtained. If the values are within the range an ON/OFF control will be performed by manipulating an electric voltage to the actuators.

Figure 12. presents the prismatic links stroke or the actuators linear displacements according to the desired target position of the moving platform. The controller was fed with a $(1,8,220)$ that produces a corresponding actuators displacement output of $1.6 \mathrm{~mm}, 8.1$ $\mathrm{mm}$ and $7.3 \mathrm{~mm}$. The figure includes both simulation and experimental results. The response is around 2 seconds. A permissible error of $\pm 0.01 \mathrm{~mm}$ was used to reduce the chattering phenomena. As it can be observed, in the figure, there is a discrepancy in experimental and simulation results. This can be attributed to the internal recovery, which is due to the formation of internal hysteresis loops when interrupting the cooling process $[15,16]$. However, the manipulator showed that any desirable position within the work space was achievable for a prescribed permissible error. Although there is a difference in response between the experimental and simulation results, the results could be considered as acceptable as long as the permissible error $\pm \alpha$ is concerned. The response obtained from the experiments was around $2 \mathrm{~s}$, which is acceptable when compared with other researchers findings. As an example is the response of the SMA actuators using self tuning fuzzy PID controller, which was designed by Kha and Ahn [17]. They obtained a response around $3 \mathrm{~s}$. Jayender et al. [18] achieved a response of $1 \mathrm{~s}$ when studying the performance of an SMA actuator control. They used an SMA wire of $0.3 \mathrm{~mm}$ diameter. The SMA wire size is also a factor that affects the response of the SMA actuators. The rate of heating and cooling are much higher for a thinner wire since the ratio of surface area to volume increases as the wire diameter is reduced.

\section{CONCLUSIONS}

A new technique of actuating a parallel platform manipulator using shape memory alloy was presented in this paper. This study shows that the new shape memory alloy actuated beam can successfully be used to provide linear displacement. A 3-UPU parallel link manipulator was developed successfully using linear SMA actuators. The control results show closeness in simulation and experimental results. The built prototype indicates the feasibility of applying SMA actuators in parallel manipulators.

\section{REFERENCES}

[1] Nam, T., Yu,C., Lee, Y., and Liu, Y., "Fabrication of NiTi alloy for proportional control", Int. J. of Appl. Electromagnetics and Mechanics, No.23, pp 9-15, (2006). 
[2] Loh, C.S, Yokoi, H., and Arai, T., "New shape memory alloy actuator: design and application in the prosthetic hand", Proc. of the 2005 IEEE Engineering in Medicine and Biology $27^{\text {th }}$ Annual Conference, Shanghai, China, pp. 6900-6903, (2005).

[3] Ikuta, K. Micro/ Miniature Shape Memory Alloy Actautor. In IEEE Robotics and Automation Society, , Los Alamitos, California, No.3, pp 2156-2161, (1990).

[4] Hirose, S., Ikuta, K. and Umetani, Y., "Development of a Shape Memory Alloy Actuator; Performance Assessment and Introduction of a New Composing Approach", Advanced Robotics, No.1, pp 3-16, (1989).

[5] Grant, D. and Hayward, V., "Constrained forced control of shape memory alloy actuators", Proceedings of the 2000 IEEE International Conference on Robotics and Automation, San Francisco, CA, pp 1314-1320, (2000).

[6] Zhong, Z.W. and Yeong, C.K., "Development of a gripper using SMA wire", Sensors and Actuators A, No.126, pp 375-381, (2006).

[7] Stewart, D., "A Platform with Six Degrees of Freedom", Proceedings of the Institute of Mechanical Engineers, London England, No.180, pp 371-386, (1965).

[8] Albus, J., Bostelman, R. and Dagalakis, N., "The NIST ROBOCRANE", Journal of Robotic Systems No.5, pp 708-724, (1993).

[9] Chirikjian, G.S., "A binary paradigm for robotic manipulators", In IEEE International Conference on Robotics \& Automation. San Diego., pp. 3063-3070, (1994).

[10] Choi, S., Park, D. and Cho, M, "Position control of a parallel link manipulator using electro-rheological valve actuators", Mechatronics, No.11, pp 157-181, (2001).

[11] Mohamed N. A. N., Haris S. M. and Khidir E. A., "Geometrical Amplification of SMA Actuator Displacement Using Externally Actuated Beam", $2^{\text {nd }}$ World Engineering Congress, Sarawak, Malaysia, pp 363-368, (2002).

[12] Latombe, J.C., Robot Motion Planning, A Kluwer Academic Publishers Series. Boston: Kluwer Academic Publishers, (1991).

[13] Elwaleed Awad Khidir, Nik Abdullah Nik Mohamed, Mohd Jailani Mohd Nor and Mohd Marzuki Mustafa, "A New Concept of a Linear Smart Actuator", Journal of Sensors and Actuators A, No.1, pp 244-249, (2007).

[14] Fu, S., Hou, Y. and Muller, I., "Thermodynamics of pseudoelasticity - an analytical approach", Acta Mechanica, No.99, pp 1-19, (1993).

[15] Choi, B., and Lee, Y., "Fast Preisach modeling method for shape memory alloy actuators using major hysteresis loops", Smart Mat. and Str. No.9, 54-59, (2004).

[16] Kha, N. and Ahn, k., "Position control of shape memory alloy actuators by using self tuning fuzzy PID controller", Proceedings of the IEEE Conference on Industrial Electronics and Applications, ICIEA 2006, Singapore, (2006).

[17] Jayender, J, Patel, R.V., Nikumb, S. \& Ostojic, $M_{\text {., " }} \mathrm{H}_{\infty}$ loop shaping controller for shape memory alloy actuators", Proc. of the $44^{\text {th }}$ IEEE Conf. on Decission and Control, and the European Control Conference, Seville, Spain, pp 653-658, (2005). 


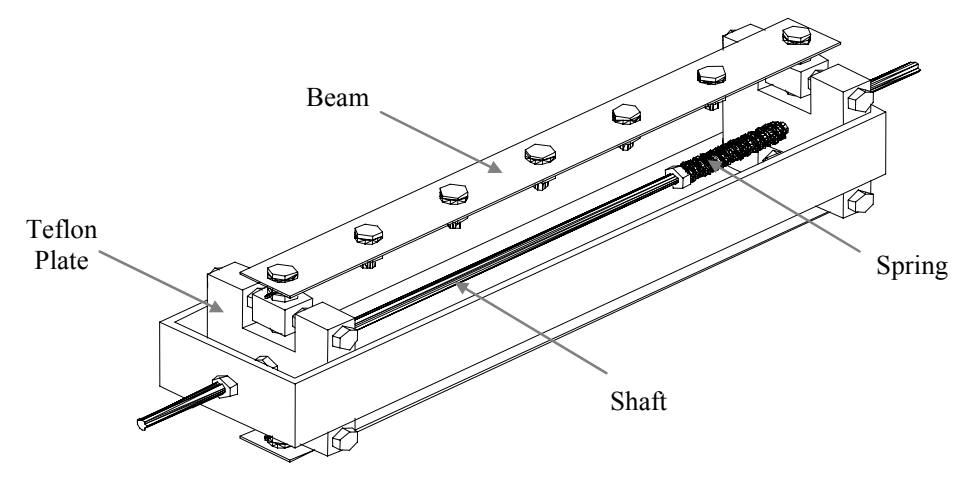

Fig. 1. 3D drawing for the shape memory alloy actuator

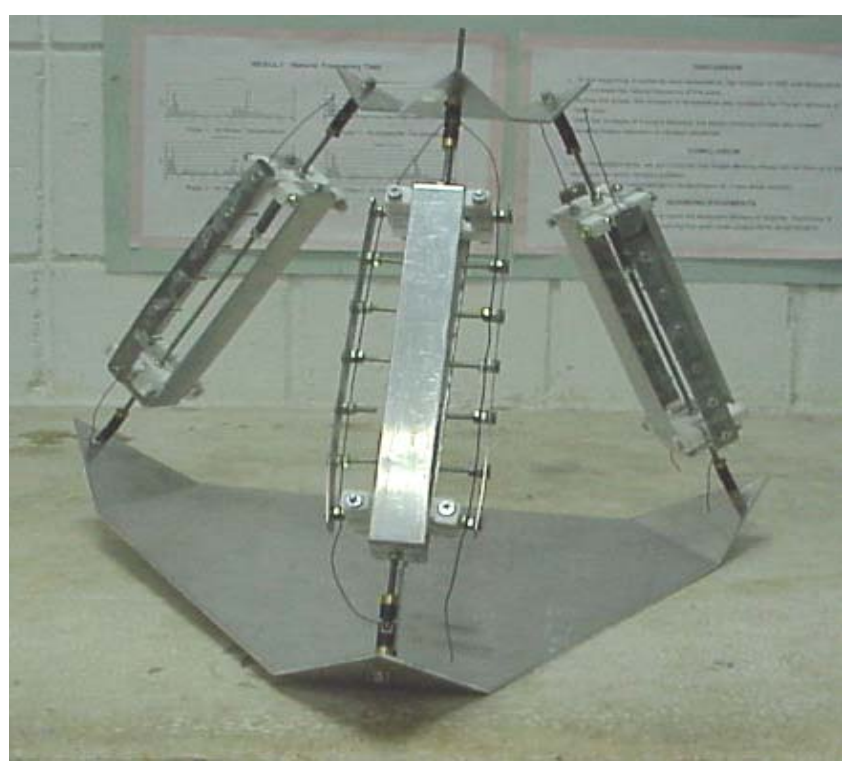

Fig. 2. Prototype of the 3-UPU parallel manipulator

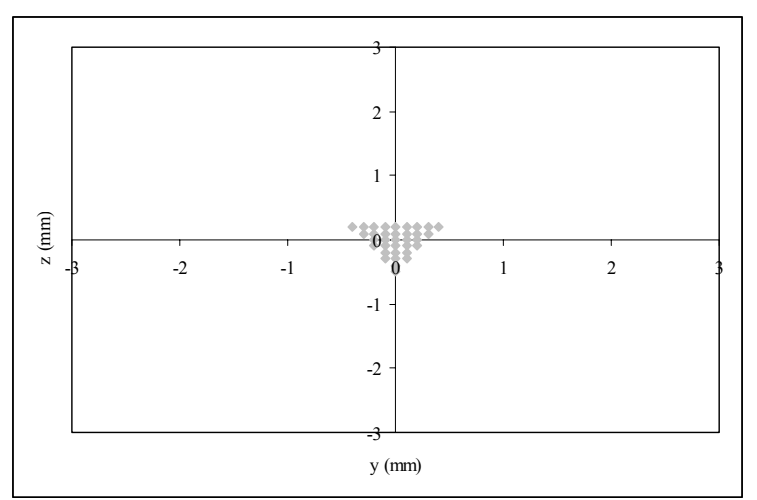

Fig. 3. Working space for joint angular constraint of $\pm \pi / 4$ at $215 \mathrm{~mm}$ plane. 


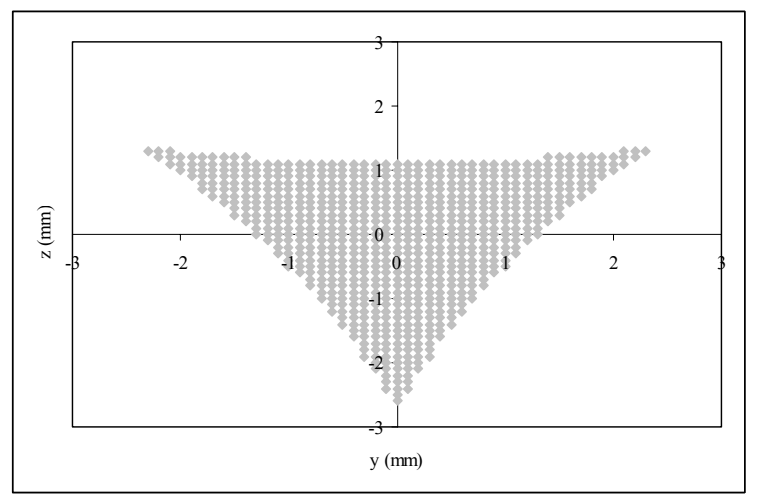

Fig. 4. Working space for joint angular constraint of $\pm \pi / 4$ at $220 \mathrm{~mm}$ plane.

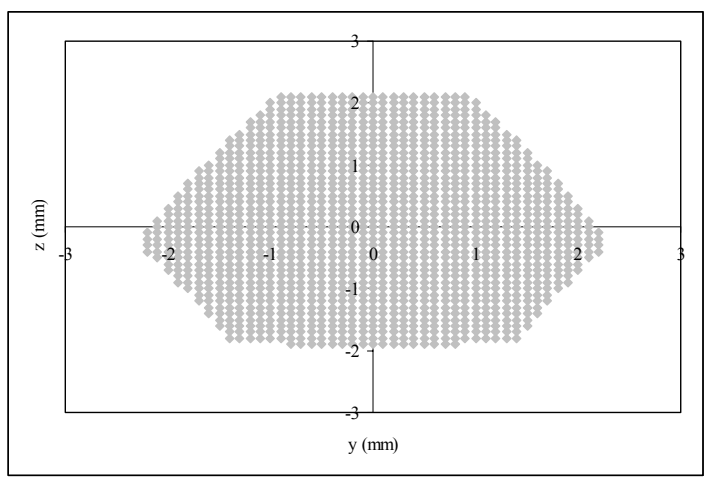

Fig. 5. Working space for joint angular constraint of $\pm \pi / 4$ at $225 \mathrm{~mm}$ plane.

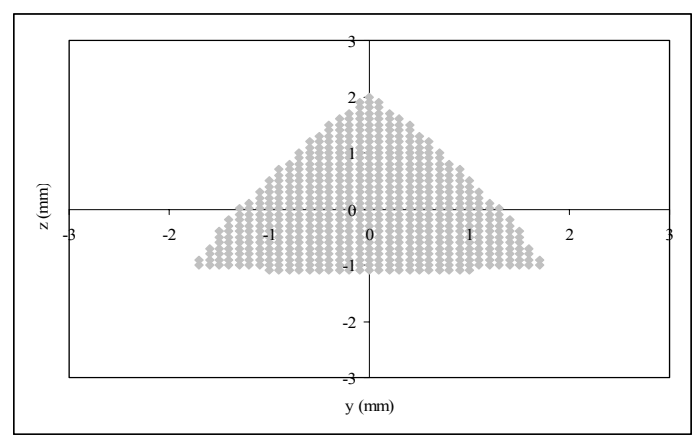

Fig. 6. Working space for joint angular constraint of $\pm \pi / 4$ at $230 \mathrm{~mm}$ plane. 


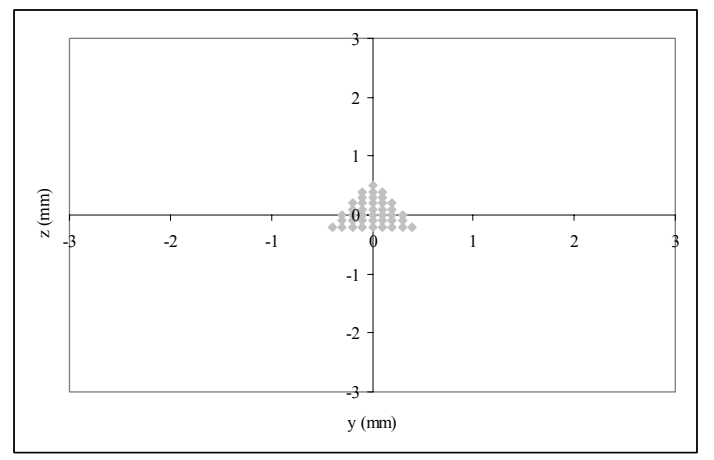

Fig. 7. Working space for joint angular constraint of $\pm \pi / 4$ at $235 \mathrm{~mm}$ plane.

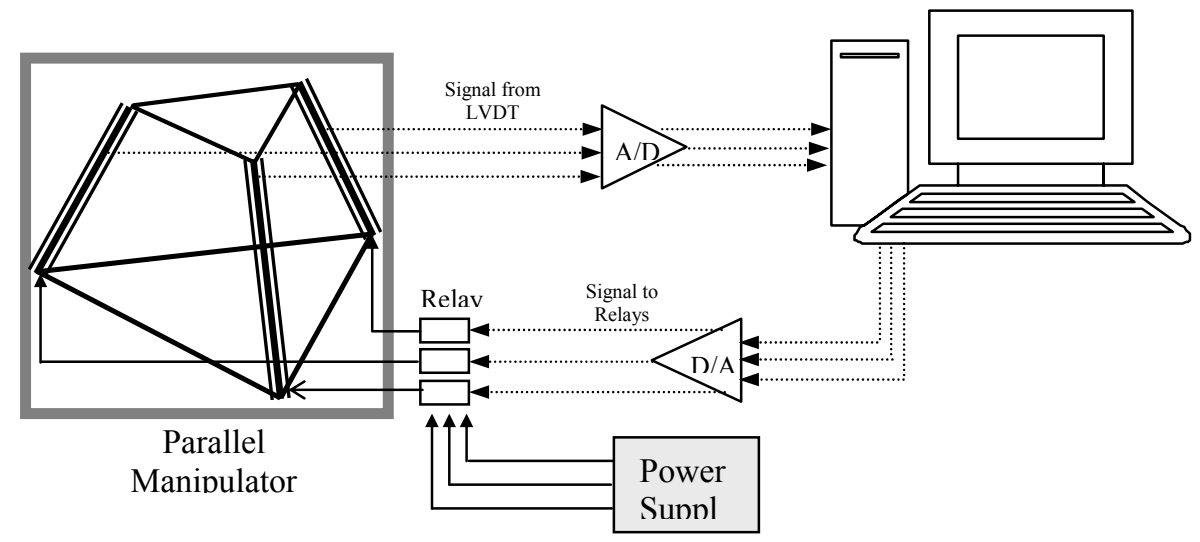

Fig. 8. Experimental setup schematic diagram for the position control of the parallel manipulator

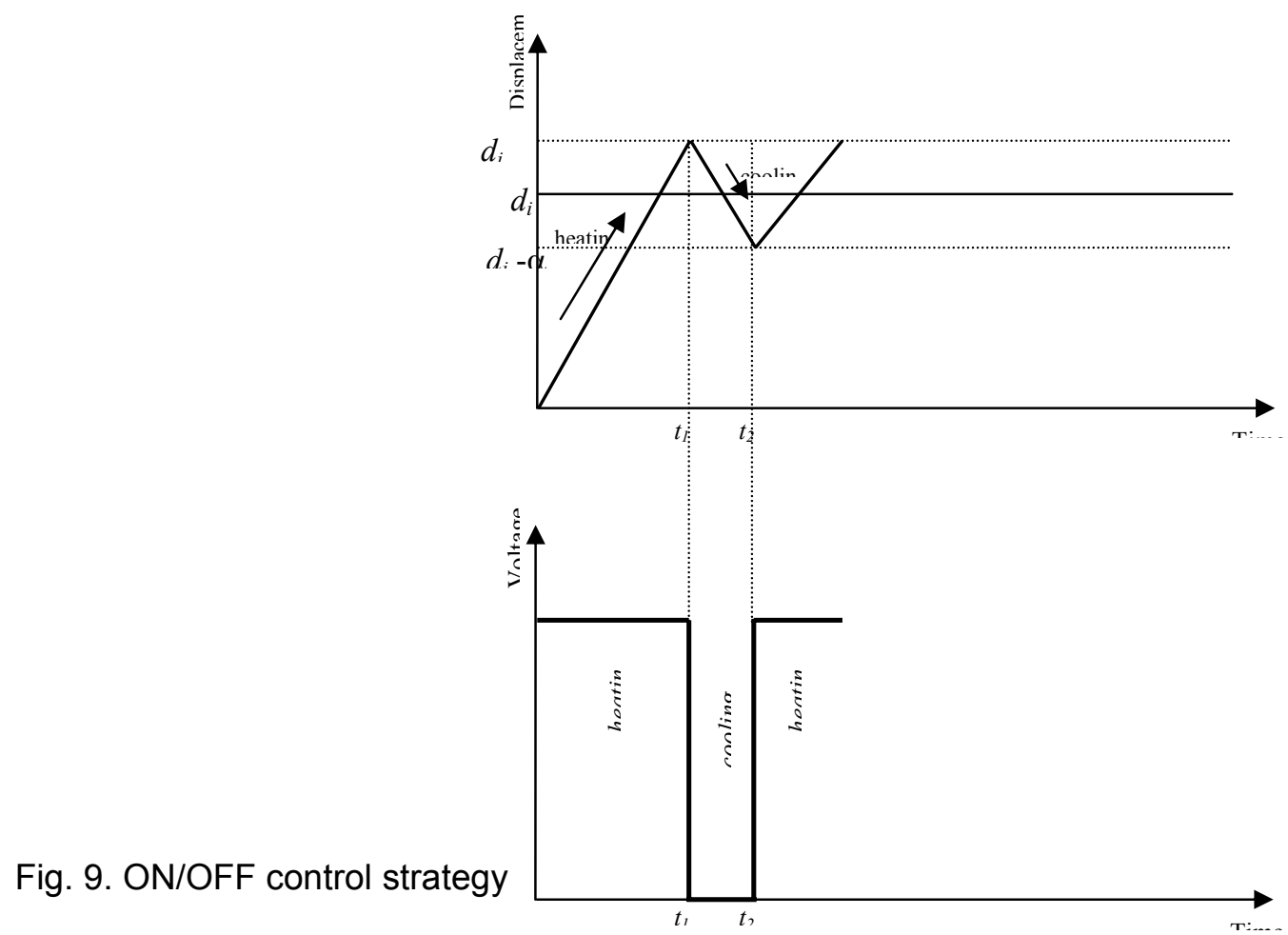




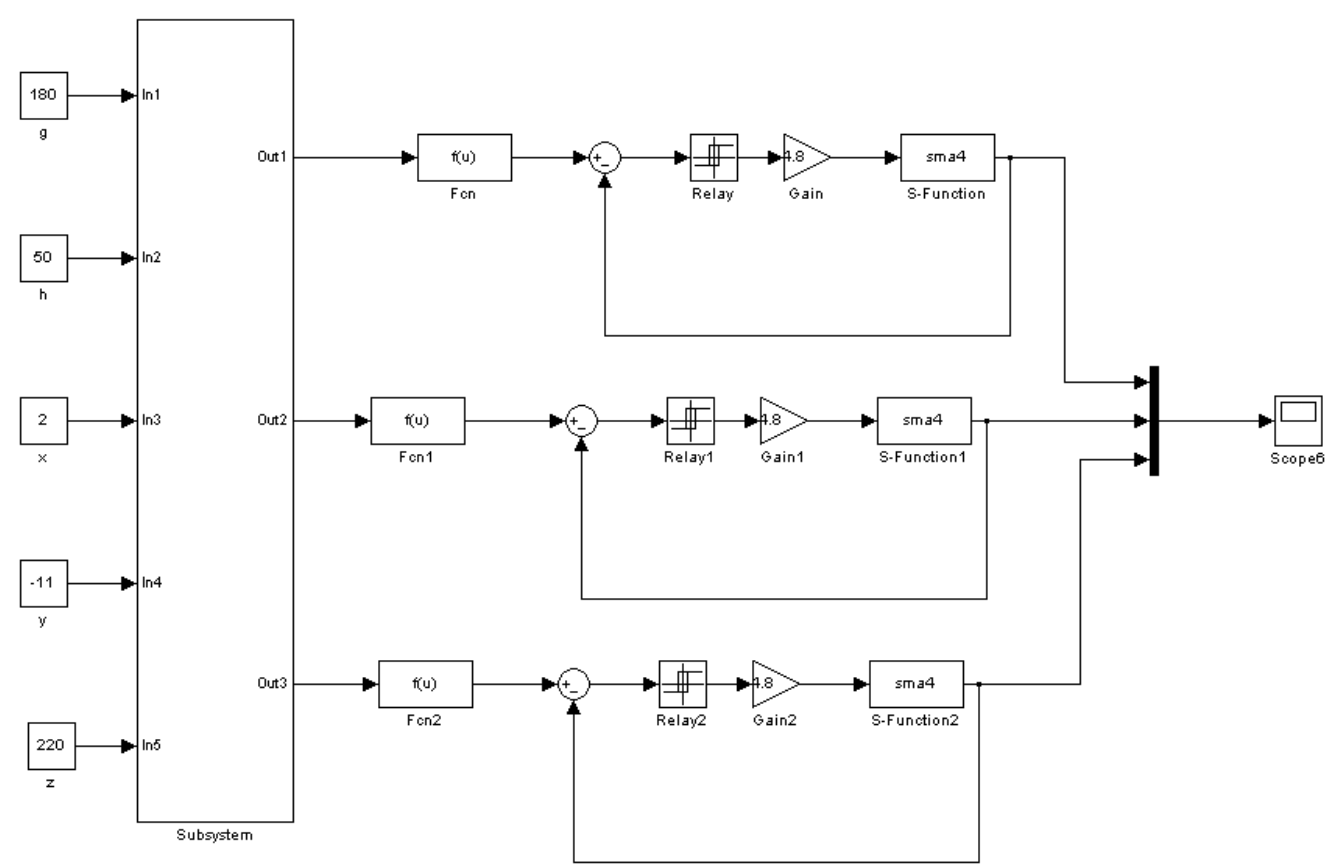

Fig. 10. Simulink diagram for the 3 links of the parallel manipulator

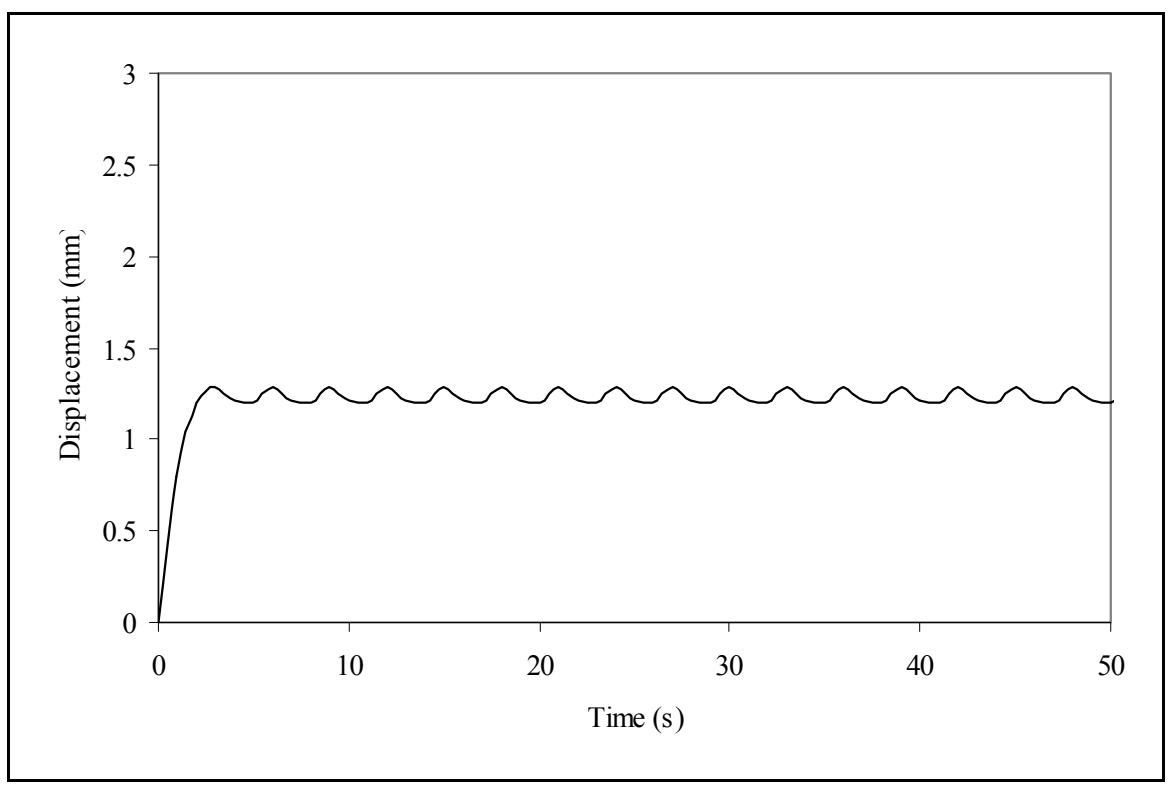

Fig. 11. The manipulator link displacement output for a given $(0,0,215)$ input 

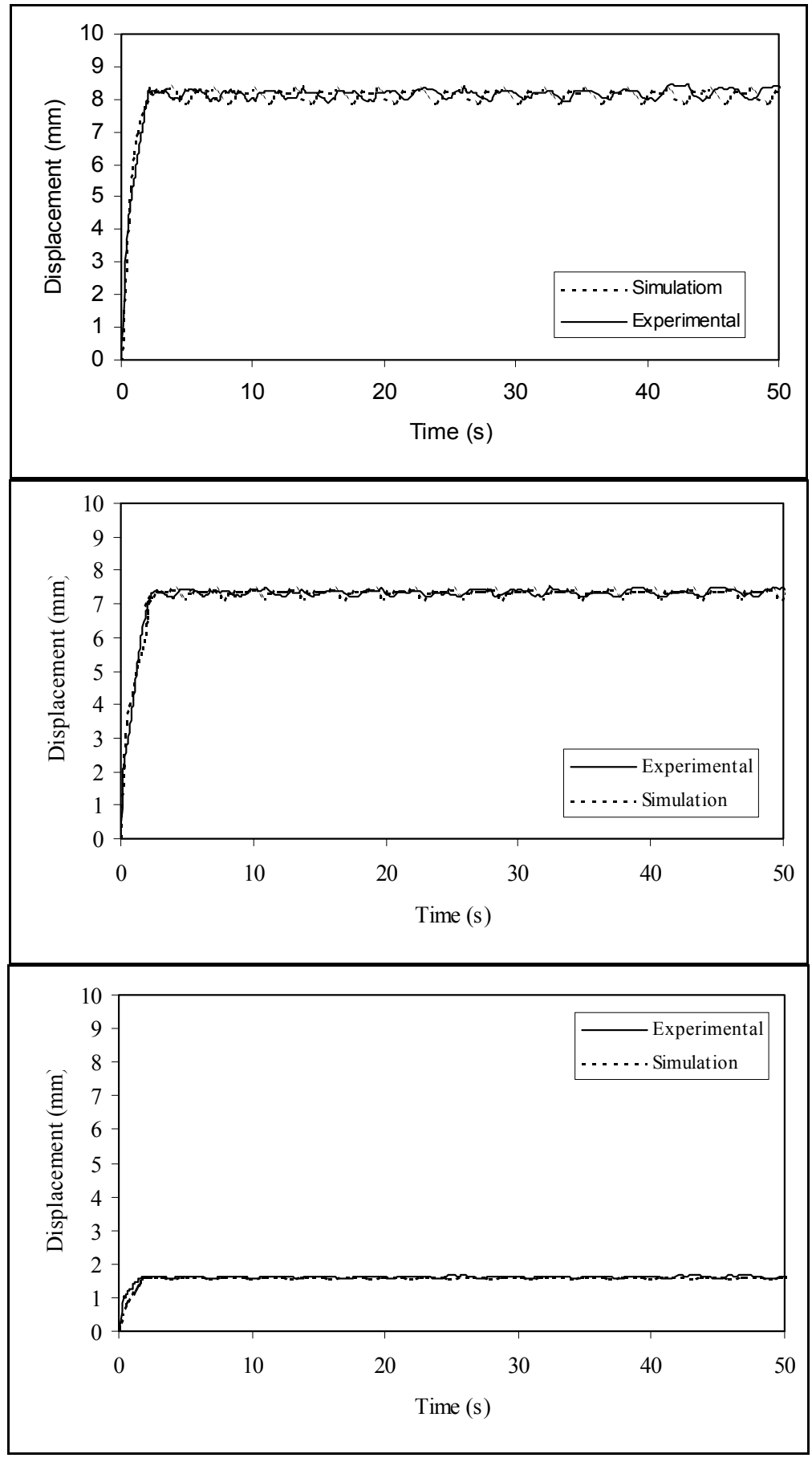

Fig. 12. Actuators displacements for $(1,8,220)$ desired position; the actuators displacements are $8.1 \mathrm{~mm}, 7.3 \mathrm{~mm}$ and $1.6 \mathrm{~mm}$ 